TITLE:

\title{
Sparing Native Upper Lobes in Living-Donor Lobar Lung Transplantation: Five Cases From a Single Center.
}

\section{$\operatorname{AUTHOR}(\mathrm{S}):$}

Aoyama, A; Chen, F; Minakata, K; Yamazaki, K; Yamada, T; Sato, M; Date, H

\section{CITATION:}

Aoyama, A ...[et al]. Sparing Native Upper Lobes in Living-Donor Lobar Lung

Transplantation: Five Cases From a Single Center.. American journal of transplantation 2015, 15(12): 3202-3207

\section{ISSUE DATE:}

2015-12

URL:

http://hdl.handle.net/2433/207649

\section{RIGHT:}

This is the accepted version of the following article: [Aoyama, A., Chen, F., Minakata, K., Yamazaki, K., Yamada, T., Sato, M. and Date, H. (2015), Sparing Native Upper Lobes in Living-Donor Lobar Lung Transplantation: Five Cases From a Single Center. American Journal of Transplantation, 15:3202-3207], which has been published in final form at http://dx.doi.org/10.1111/ajt.13357. This article may be used for non-commercial purposes in accordance with Wiley Terms and Conditions for Self-Archiving.; The full-text file will be made open to the public on 20 November 2016 in accordance with publisher's 'Terms and Conditions for Self-Archiving'.; This is not the published version. Please cite only the published version;; この論文は出版社版でありません。引用の際には出版社版をご確認ご利用ください。 
Sparing Native Upper Lobes in Living-donor Lobar Lung Transplantation: Five Cases from a Single Center

Aoyama $\mathrm{A}^{\mathrm{a}}$, Chen $\mathrm{F}^{\mathrm{a}}$, Minakata $\mathrm{K}^{\mathrm{b}}$, Yamazaki $\mathrm{K}^{\mathrm{b}}$, Yamada $\mathrm{T}^{\mathrm{a}}$, Sato $\mathrm{M}^{\mathrm{a}}$, Date $\mathrm{H}^{\mathrm{a}}$

${ }^{\mathrm{a}}$ Thoracic Surgery, ${ }^{\mathrm{a}}$ Cardiovascular Surgery, Graduate School of Medicine, Kyoto University, Kyoto, Japan

\section{Corresponding author contact information:}

Hiroshi Date, MD, PhD

Department of Thoracic Surgery, Graduate School of Medicine, Kyoto University

54 Shogoin-Kawara-cho, Sakyo-ku, Kyoto, 606-8507, Japan

TEL: $+81-75-751-4975$

Fax: $+81-75-751-4974$

E-mail: hdate@kuhp.kyoto-u.ac.jp

Word counts: Abstract: 190 words, text: 2457 words

Type of submission: brief communication

\section{Running title: Sparing Upper Lobes in Lobar Lung Transplantation}

Keywords: Living donor, lung transplantation, native, small graft, spare

\section{Abbreviation list:}

$\mathrm{BO}$, bronchiolitis obliterans

$\mathrm{CPB}$, cardiopulmonary bypass

CHP, chronic hypersensitivity pneumonitis

$\% \mathrm{DLCO}$, diffusing capacity of the lung for carbon monoxide

ECMO, extracorporeal membrane oxygenation

FVC, forced vital capacity

HRCT, high-resolution computed tomography 
ICU, intensive care unit

IIP, idiopathic interstitial pneumonia

IPAH, idiopathic pulmonary arterial hypertension

LDLLT, living-donor lobar lung transplantation

PAP, pulmonary artery pressure

PGD, primary graft dysfunction

PVR, pulmonary vascular resistance

Tc99-MAA, Tc-99 macroaggregated albumin 


\begin{abstract}
Living-donor lobar lung transplantation (LDLLT) is indicated for rapidly deteriorating patients, and the total volume of 2 lower lobe grafts must be sufficient for the recipient. To rescue patients with small lobar grafts, we performed 5 LDLLTs sparing native upper lobes. This strategy was used when upper lobes or segments were preoperatively less impaired. There were no hospital deaths. Extracorporeal circulation time and operative time were similar to those of conventional LDLLTs. The length of intensive care unit stay was also similar. Late complications attributed to the spared lungs were airway infection in one recipient and pneumothorax in two but they were successfully managed. All recipients were discharged without supplemental oxygen. The spared lung volumes measured by volumetry did not change after LDLLT. Lung perfusion scintigraphy performed at one year showed remaining perfusion in the spared lungs, although much less than in the grafts. These results suggested that the spared lobes kept adequate space in the thoracic cavity and kept functioning to a limited extent. The new "lobar-sparing" strategy appears feasible and effective in LDLLT using small grafts for selected patients when the upper lobes or segments are less impaired.
\end{abstract}

\title{
Introduction
}

For rapidly deteriorating patients, living-donor lobar lung transplantation (LDLLT) was developed by Starnes and his colleagues, and has yielded acceptable results (1-3). LDLLTs account for approximately $40 \%$ of all lung transplants in Japan, where the average waiting time for a deceased donor lung is more than 2 years (4). In LDLLT, the total forced vital capacity (FVC) of the 2 lower lobar grafts must be $50 \%$ of the predicted $\mathrm{FVC}$ of the recipient calculated from height, age, and sex (5). Therefore, transplants from short donors to tall recipients have been generally contraindicated for LDLLT.

A native lobe-sparing lobar transplant technique was reportedly feasible in a canine 
model (6). The first unilateral native lobe-sparing lobar lung transplantation for emphysema was reported in 2006 (7). We also reported a successful case of a bilateral native lobe-sparing LDLLT in $2011(8,9)$, and to date, this novel strategy has been applied to 5 LDLLTs that would otherwise be very challenging because of small grafts. This procedure was initially aimed to reserve as much lung function as possible and to minimize intrathoracic dead space to avoid complications such as pleuritis and graft hyperinflation (10). Herein, we report the short- and mid-term results of those 5 LDLLTs with the native upper lobes or segments spared.

\section{Patients and Methods}

Between August 2008 and October 2014, 49 patients underwent 51 LDLLTs, including 11 single lobar transplantations, at Kyoto University Hospital. Among 40 bilateral lobar transplantations, 35 LDLLTs were performed after bilateral pneumonectomy (Normal Group), and 5 LDLLTs were performed with the native upper lobes or segments spared because the lobar grafts were thought to be too small (Sparing Group). The recipients in the Sparing Group were all adult men. The unilateral upper lobe or segment was spared in 2 patients and bilateral upper lobes or segments were spared in 3 patients. The indications for LDLLTs in the Sparing Group were idiopathic interstitial pneumonia (IIP) in 2 recipients and bronchiolitis obliterans (BO), chronic hypersensitivity pneumonitis (CHP), and idiopathic pulmonary artery hypertension (IPAH) in 1 recipient, respectively. They were all rapidly deteriorating and unlikely to survive the long waiting time for deceased donors. The predicted $\% \mathrm{FVC}$ calculated from the donor FVC was $40 \%$ to $58 \%$ of the predicted $\mathrm{FVC}$ of the recipient, with an average of $50 \%$, which was significantly lower than $69 \%$ in the Normal Group. A summary of the 5 cases, including the results of preoperative pulmonary function tests and 6-minute walk tests, is shown in Table 1. The Ethics Committee of Kyoto University Hospital approved the protocol, and informed consent was obtained.

We applied the "sparing" strategy according to the following criteria: 
- The post-transplant predicted FVC from 2 grafts was less than $60 \%$ of the recipient's predicted FVC.

- The lungs were not infected.

- The interlobar fissure was well developed.

- The lungs were heterogeneously impaired with the upper lobe(s) less impaired on high-resolution computed tomography (HRCT) or better perfused on perfusion scintigraphy. A preoperative CT scan of Patient 3 shows the left upper lobe to be less affected (Figure 1A).

\section{Surgical technique}

All bilateral LDLLTs were performed with standard cardiopulmonary bypass (CPB) or extracorporeal membrane oxygenation (ECMO) through a clamshell incision. CPB or ECMO was initiated when the hemodynamics became unstable or dissection of the hilar areas was completed. ECMO was preferred for the recent cases, because it reduces bleeding risk compared to CPB. The procedure in the Sparing Group was basically similar to that in conventional LDLLT (5), except that the pulmonary vein was anastomosed to the lower pulmonary vein, the pulmonary artery to the interlobar artery, and the bronchus distally to the $2^{\text {nd }}$ carina (Figure 2). If the lingular segment was affected, it was resected with a surgical stapler before heparinization to avoid bleeding from the staple line. After ECMO was initiated with an activating coagulation time between 150-200 seconds (11), the right middle and lower lobe was removed, and then the right lower lobar graft, flushed with cooled ET-Kyoto solution (Otsuka Pharmaceuticals, Tokushima, Japan), was implanted and reperfused. Then, following left lower lobectomy, the left lobar graft was sequentially implanted and reperfused. Weaning from ECMO was completed without difficulty.

\section{Postoperative management and follow-up}


Postoperative management was similar to that of the Normal Group (5).

Immunosuppression consisted of triple-drug therapy with tacrolimus or cyclosporine, mycophenolate mofetil, and corticosteroids without induction therapy. Cytomegalovirus prophylaxis with ganciclovir was administered to all recipients for more than 3 mo. Pulmonary function tests were performed before the transplant and at 3, 6, and 12 mo. We evaluated the spared lobe volumes at 3, 6, and 12 mo using 3D-CT volumetry (12). 3D-CT volumetry can be applied to measure lung volume, and the protocol was described elsewhere $(12,13)$. Briefly, CT images were obtained during a single respiratory pause at the end of maximum inspiratory effort using a multidetector Aquilion 64 CT scanner (Toshiba Medical Systems, Tochigi, Japan). The CT images were transported to an AZE VirtualPlace Lexus workstation (AZE Co., Ltd., Tokyo, Japan), and the spared lobe or segment volume was calculated. The spared lung perfusion was also evaluated using Tc-99 macroaggregated albumin (Tc99-MAA) lung perfusion scintigraphy. Imaging with a gamma camera was started immediately after Tc-99-MAA injection. The right or left lateral view images were used for evaluation because the lateral view most easily isolates the spared upper lobe or segment and the transplanted lower lobe.

\section{Statistical analysis}

Statistical analyses were performed using the JMP 11 software (SAS Institute, Cary, NC). All values were presented as the mean and compared by unpaired $t$ test. A $p$-value $<0.05$ was considered significant.

\section{Results}

Perioperative course: There were no operative or hospital deaths. Extracorporeal circulation time and operative time $(224 \mathrm{~min}, 472 \mathrm{~min})$ were similar to those of the Normal Group (249 min, 497 min). No Sparing Group recipients needed postoperative ECMO support for primary graft dysfunction (PGD), while 3 of 35 needed ECMO in the Normal Group. The 
length of intensive care unit (ICU) stay of the Sparing Group was also similar to that of the Normal Group (12.2 vs. 13.0 days). The preoperative pulmonary artery pressure (PAP) of Patient 5 with IPAH was $70 / 36 \mathrm{mmHg}$ on maximal medication, including intravenous inotropic agents in addition to epoprostenol, $40 \mathrm{ng} / \mathrm{kg} / \mathrm{min}$, and PAP immediately after reperfusion was 44/26 mmHg. Right heart catheterization carried out on postoperative day 35 confirmed normal PAP, 24/6 (mean 13) $\mathrm{mmHg}$, cardiac index, $3.6 \mathrm{~L} / \mathrm{min} / \mathrm{m}^{2}$, and pulmonary vascular resistance (PVR), 145 dynes $\cdot \mathrm{sec} / \mathrm{cm}^{5}$.

Mid-term outcome: Late complications attributed to the spared upper lobes or segments, which were all conservatively managed, included airway co-infection with Aspergillus and cytomegalovirus in Patient 2 at 5 mo post-LDLLT and pneumothorax in Patients 2 and 4 at 22 and 14 mo, respectively. All patients were discharged without supplemental oxygen. Early postoperative bronchoscopies did not show any stenosis or dehiscence at anastomoses. However, Patient 2 developed late-onset segmental bronchial stenosis that required repeated laser ablations and balloon dilations. Follow-up pulmonary function tests indicated that percent predicted forced expiratory volume in 1 second improved by LDLLT, except in Patient 5, who was not preoperatively examined, and in Patient 2, who experienced remarkable improvement in symptoms and oxygenation (Figure 3A). The average percent predicted diffusing capacity of the lung for carbon monoxide (\%DLCO) in the Sparing Group at 1 year was $62.8 \%$, which was significantly higher than that in the Normal Group (46.0\% of the 19 available patients, excluding pediatric patients; $P=0.019$ ). The median 6-minute walk distance at 1 year was $610 \mathrm{~m}$ (range, 543-678 m), suggesting that the patients had good recovery of their physical function. One recipient with IIP (Patient 2) died of chronic graft dysfunction at 3.3 years, and the other 4 are alive at $1,1.6,1.7$, and 5.4 years with good performance status.

The fibrosis of the spared lungs in the patients with IIP and CHP did not remarkably 
progress after LDLLT in HRCT scan images, as seen in the CT scan of Patient 3 (Figure 1). No remarkable progression of hyperinflation was observed in the spared upper lobes of Patient 1 with $\mathrm{BO}$, either (8). Although a slight decline in spared lung volumes, measured by 3D-CT volumetry, was observed immediately after LDLLT in Patients 2 and 4 with the decreased lung compliance characteristics of restrictive diseases, the volumes of all 8 spared lungs did not significantly change from 3 months to 1 year post-LDLLT, suggesting that the spared lungs kept filling dead space (Figure 3B). Tc99-MAA lung perfusion scintigraphy performed at 1 year showed remaining perfusion in the spared lungs, although much less than in the grafts, except in the spared upper lobes showing quite limited perfusion of the Patient 5 with IPAH (Figure 4).

\section{Discussion}

LDLLT is a therapeutic option for rapidly deteriorating patients with end-stage lung or pulmonary vascular diseases who will not survive until deceased donor lungs are allocated. However, size matching sometimes limits its chance for large recipients. To rescue such patients with small lobar grafts, we performed 5 LDLLTs with the native upper lobes spared and evaluated the short- and mid-term outcomes. A canine model and a few cases have been reported, convincing us that sparing the upper lobes in LDLLT is a feasible option, at least in short time periods (6-9).

The candidates for this novel strategy must meet several conditions. First, the absence of infection in the lung must be confirmed by culture of airway secretions or from serological or radiological studies. Second, the interlobar fissure must be well developed on CT scans to avoid air leakage or bleeding at surgery. Most importantly, the upper lobes or segments must be less impaired than the lower lobes. From this point of view, usual interstitial pneumonia, most common among patients with IIP, could be an ideal indication because of the basal involvement on HRCT (14).

For Patient 5 with IPAH with small lobar grafts amounting to only $40 \%$ of the 
predicted FVC for the recipient, we preoperatively evaluated the perfusion scintigraphy and found that the upper lungs were better perfused, which is consistent with the report suggesting that the upper lungs in IPAH are dominantly perfused but less ventilated (15). The patient was bed-bound and receiving continuous intravenous epoprostenol, dobutamine, and milrinone. After LDLLT with sparing the native bilateral upper lobes, the PAP and PVR were normalized, with only limited perfusion remaining in the upper lobes at 1 year after LDLLT, which indicated almost no shunting. However, if PAP is increased for some reason, the grafts might be perfused to some extent, which could be helpful in preventing lung edema and right heart failure, although possibly harmful in oxygenation. This putative mechanism should possibly be in operation in the perioperative period to avoid severe PGD.

One may consider this strategy bothersome because of the possibly complicated procedures. However, excellent exposure through the clamshell approach enables us to perform bilateral lobectomies followed by implantation without prolonging extracorporeal circulation or operative times compared to the Normal Group. In addition, the length of ICU stay was also similar, suggesting that the perioperative course was not more complicated despite the difference in surgical procedure and graft size.

We originally expected the spared lungs to keep functioning after LDLLT and to provide lung volume to minimize dead space. Regarding lung volume, CT volumetry indicated that the spared lungs actually provided stable volume in the chest cavity at least for 1 year, regardless of disease type - restrictive, obstructive, or pulmonary vascular. Thus, the spared lobes reduced intrathoracic dead space and provided adequate chest cavity for small grafts. On the other hand, the spared lung perfusion remained at 1 year was quite limited as shown in Figure 4 . The fact that the average $\%$ DLCO in the Sparing Group at 1 year was significantly higher than that in the Normal Group might also indicate that the severe V/Q mismatch or shunt was not present. Patients with low pulmonary PVR or very high $\mathrm{O}_{2}$ requirements, such as those undergoing nasal high-flow therapy, might develop postoperative hypoxia due 
to shunting when native lobes are spared. Although preoperative PVR was not measured, except in Patient 5, the PVR of the spared lobes should be higher than that of the totally normal lung grafts. The patients preoperatively required supplemental oxygen of only 1 to 2 $\mathrm{L} / \mathrm{min}$ at rest, or up to $5 \mathrm{~L} / \mathrm{min}$ at exercise, although they were deteriorating and unlikely to survive the long waiting time for deceased donors. This might be the reason why all the patients did not require supplemental oxygen postoperatively, in addition to the fact that the spared lungs were less impaired compared to the rest of the native lungs.

. Although it is unclear how much the spared lungs were perfused immediately after graft reperfusion, none of the Sparing Group patients needed postoperative ECMO support for severe PGD despite the small grafts, a risk factor for PGD (16). The spared lungs might serve as a reserve of blood bed after reperfusion, at least in the early postoperative period.

Adverse events include pneumothorax and presumably de novo airway infection, and they were conservatively treated. In addition, native lungs with IIP or patients with smoking history are at risk of cancer. Therefore, as in single lung transplantation, the spared lungs should be carefully followed up. These complications did not directly lead to mortality, and 4 of 5 patients are alive at $1,1.6,1.7$, and 5.4 years after LDLLT.

In conclusion, the new lobar-sparing strategy appears to be feasible and effective in LDLLT using small-for-size grafts for selected patients when the upper lobes or segments are less impaired, although a longer follow-up and further accumulation of cases are necessary.

\section{Disclosure}

This report is not supported by any funds. The authors of this manuscript have no conflicts of interest to disclose as described by the American Journal of Transplantation. 


\section{Acknowledgments}

We thank Dr. Kazuo Chin for the respiratory management of the patients and Dr.

Hideyuki Kinoshita for the evaluation and management of pulmonary hypertension in the patients. 
Figure legends

Table 1

Characteristics of the recipients and the donors, preoperative pulmonary function test and 6 -minute walk test of the recipients, predicted postoperative $\% \mathrm{FVC}$ without sparing native lungs, and spared lobes/segments. BO, bronchiolitis obliterans; CHP, chronic hypersensitivity pneumonitis; IIP, idiopathic interstitial pneumonia; IPAH, idiopathic pulmonary arterial hypertension; UL, upper lobe; Seg., segment; NT, not tested because the patient was too sick.

Figure 1

Preoperative (A) and 1-year postoperative (B) CT scan images of Patient 3, in whom the left upper segment was spared. The left upper lobe was preserved, and no remarkable progression was observed at 1 year after transplantation in the spared lobe.

Figure 2

Sparing native upper lobes in living-donor lobar lung transplantation. Bilobectomy and left lower lobectomy are performed in the recipient, and lower lobar grafts are implanted.

Figure 3

(A) Pre- and postoperative percent predicted forced expiratory volume in 1 second. The patient with IPAH (Patient 5) did not receive a pulmonary function test before transplantation. (B) Spared lung volumes assessed by 3D-CT volumetry. The dotted lines mean resection of the lingular segment. BO, bronchiolitis obliterans; CHP, chronic hypersensitivity pneumonitis; IIP, idiopathic interstitial pneumonia; IPAH, idiopathic pulmonary arterial hypertension. 
Figure 4

The right and left lateral view images of Tc-99 macroaggregated albumin lung perfusion scintigraphy at 1 year after LDLLT. The areas surrounded by dotted lines indicate the spared lungs. BO, bronchiolitis obliterans; CHP, chronic hypersensitivity pneumonitis; IIP, idiopathic interstitial pneumonia; IPAH, idiopathic pulmonary artery hypertension; A, anterior; $\mathrm{P}$, posterior.

1. Starnes VA, Barr ML, Cohen RG. Lobar transplantation. Indications, technique, and outcome. The Journal of thoracic and cardiovascular surgery. 1994;108(3):403-10; discussion $10-1$

2. Starnes VA, Bowdish ME, Woo MS, Barbers RG, Schenkel FA, Horn MV, et al. A decade of living lobar lung transplantation: recipient outcomes. The Journal of thoracic and cardiovascular surgery. 2004;127(1):114-22.

3. Date H, Sato M, Aoyama A, Yamada T, Mizota T, Kinoshita H, et al. Living-donor lobar lung transplantation provides similar survival to cadaveric lung transplantation even for very ill patientsdagger. European journal of cardio-thoracic surgery : official journal of the European Association for Cardio-thoracic Surgery. 2014.

4. Sato M, Okada Y, Oto T, Minami M, Shiraishi T, Nagayasu T, et al. Registry of the 
Japanese Society of Lung and Heart-Lung Transplantation: official Japanese lung transplantation report, 2014. General thoracic and cardiovascular surgery. 2014;62(10):594-601.

5. Date H, Aoe M, Nagahiro I, Sano Y, Andou A, Matsubara H, et al. Living-donor lobar lung transplantation for various lung diseases. The Journal of thoracic and cardiovascular surgery. 2003;126(2):476-81.

6. Sugimoto S, Date H, Sugimoto R, Aoe M, Sano Y. Bilateral native lung-sparing lobar transplantation in a canine model. The Journal of thoracic and cardiovascular surgery. 2006;132(5):1213-8.

7. Yamane M, Okutani D, Sugimoto S, Toyooka S, Aoe M, Okazaki M, et al. Native lung-sparing lobar transplantation for pulmonary emphysema. The Journal of heart and lung transplantation : the official publication of the International Society for Heart Transplantation. 2008;27(9):1046-9.

8. Fujinaga T, Bando T, Nakajima D, Sakamoto J, Chen F, Shoji T, et al. Living-donor lobar lung transplantation with sparing of bilateral native upper lobes: a novel strategy. The Journal of heart and lung transplantation : the official publication of the International Society for Heart Transplantation. 2011;30(3):351-3. 
9. Chen F, Fujinaga T, Bando T, Date H. Pulmonary function of individual lung lobes after complex living-donor lobar lung transplantation using inspiratory and expiratory three-dimensional computed tomographic volumetry. Interactive cardiovascular and thoracic surgery. 2012;15(6):1077-9.

10. Haddy SM, Bremner RM, Moore-Jefferies EW, Thangathurai D, Schenkel FA, Barr ML, et al. Hyperinflation resulting in hemodynamic collapse following living donor lobar transplantation. Anesthesiology. 2002;97(5):1315-7.

11. Zuercher AJ, Inci I, Benden C, Fretz G, Bechir M, Boehler A, et al. Intra-operative extracorporeal membrane oxygenation use in pediatric lung transplantation--the Zurich experience. Pediatric transplantation. 2013;17(8):800-5.

12. Chen F, Kubo T, Yamada T, Sato M, Aoyama A, Bando T, et al. Adaptation over a wide range of donor graft lung size discrepancies in living-donor lobar lung transplantation. American journal of transplantation : official journal of the American Society of Transplantation and the American Society of Transplant Surgeons. 2013;13(5):1336-42.

13. Chen F, Fujinaga $\mathrm{T}$, Shoji $\mathrm{T}$, Yamada $\mathrm{T}$, Nakajima D, Sakamoto J, et al. Perioperative assessment of oversized lobar graft downsizing in living-donor lobar lung 
transplantation using three-dimensional computed tomographic volumetry. Transplant international : official journal of the European Society for Organ Transplantation. 2010;23(9):e41-4.

14. Raghu G, Collard HR, Egan JJ, Martinez FJ, Behr J, Brown KK, et al. An official ATS/ERS/JRS/ALAT statement: idiopathic pulmonary fibrosis: evidence-based guidelines for diagnosis and management. American journal of respiratory and critical care medicine. 2011;183(6):788-824.

15. Suga K, Tokuda O, Okada M, Koike M, Iwanaga H, Matsunaga N. Assessment of cross-sectional lung ventilation-perfusion imbalance in primary and passive pulmonary hypertension with automated V/Q SPECT. Nuclear medicine communications. 2010;31(7):673-81.

16. Eberlein M, Arnaoutakis GJ, Yarmus L, Feller-Kopman D, Dezube R, Chahla MF, et al. The effect of lung size mismatch on complications and resource utilization after bilateral lung transplantation. The Journal of heart and lung transplantation : the official publication of the International Society for Heart Transplantation. 2012;31(5):492-500. 


\begin{tabular}{|c|c|c|c|c|c|c|c|}
\hline \multirow{2}{*}{ Patient } & \multirow{2}{*}{ Disease } & \multirow{2}{*}{$\begin{array}{l}\text { Age } \\
\text { sex }\end{array}$} & \multirow{2}{*}{$\begin{array}{l}\text { Height } \\
(\mathrm{cm})\end{array}$} & Right donor & Left donor & \multirow{2}{*}{$\begin{array}{l}\text { Predicted } \\
\text { postoperative } \\
\text { \%FVC }\end{array}$} & \multirow{2}{*}{ Spared } \\
\hline & & & & Age, relation, height & Age, relation, height & & \\
\hline 1 & BO & $44, \mathrm{M}$ & 170 & 45, sister, 154 & 42, wife, 154 & 46 & $\begin{array}{c}\text { Rt UL } \\
\text { Lt upper Seg. }\end{array}$ \\
\hline 2 & IIP & $61, M$ & 160 & 39 , daughter, 160 & 30 , son, 160 & 52 & Rt UL \\
\hline 3 & IIP & $54, \mathrm{M}$ & 163 & 24, son, 177 & 58, wife, 161 & 58 & Lt upper Seg. \\
\hline 4 & $\mathrm{CHP}$ & $56, \mathrm{M}$ & 171 & 22, son, 172 & 25, son, 171 & 56 & Bilateral ULs \\
\hline 5 & IPAH & $21, \mathrm{M}$ & 170 & 57, father, 171 & 49, mother, 169 & 40 & Bilateral ULs \\
\hline
\end{tabular}


Figure 1
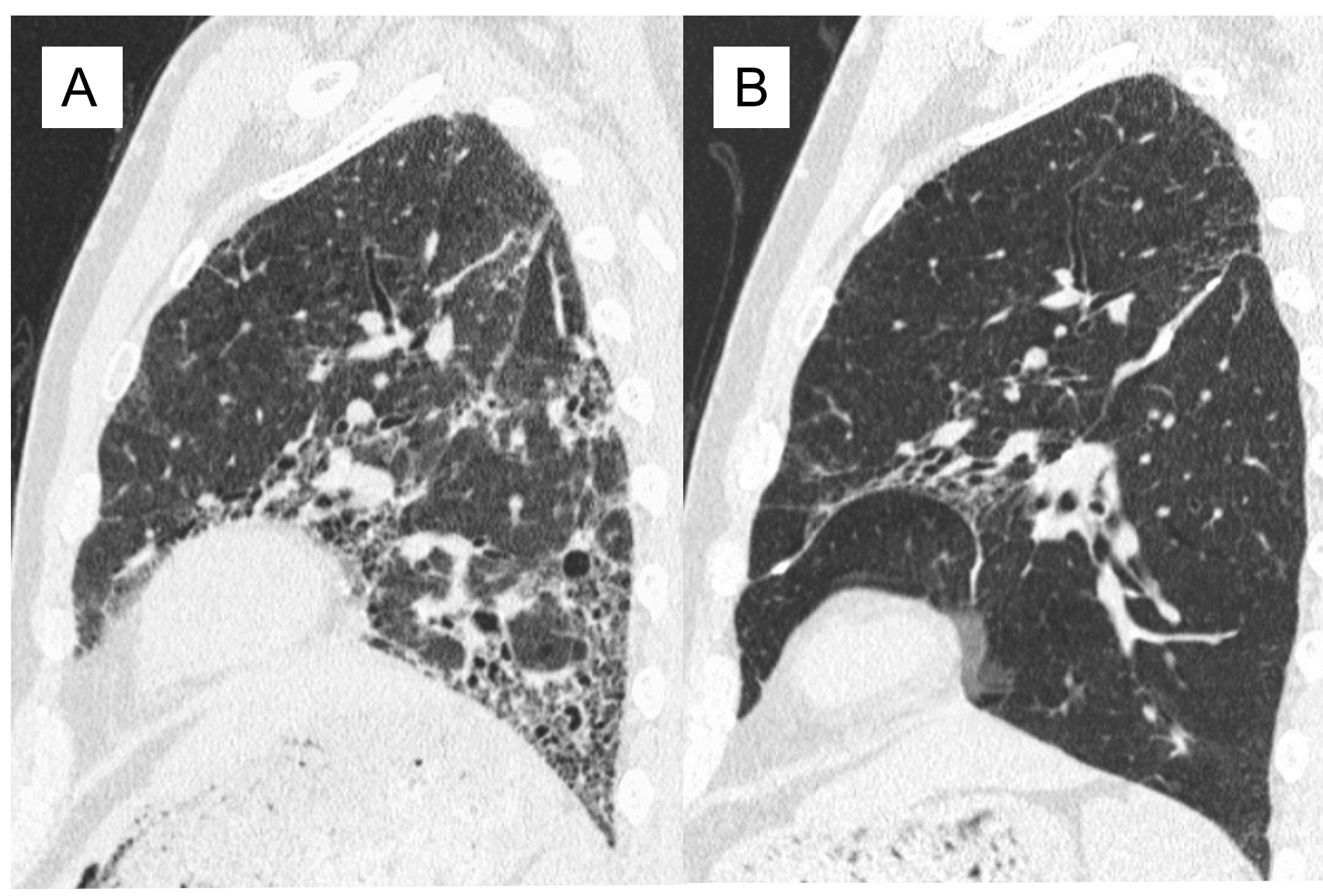
Figure 2

Recipient
"Recisposition
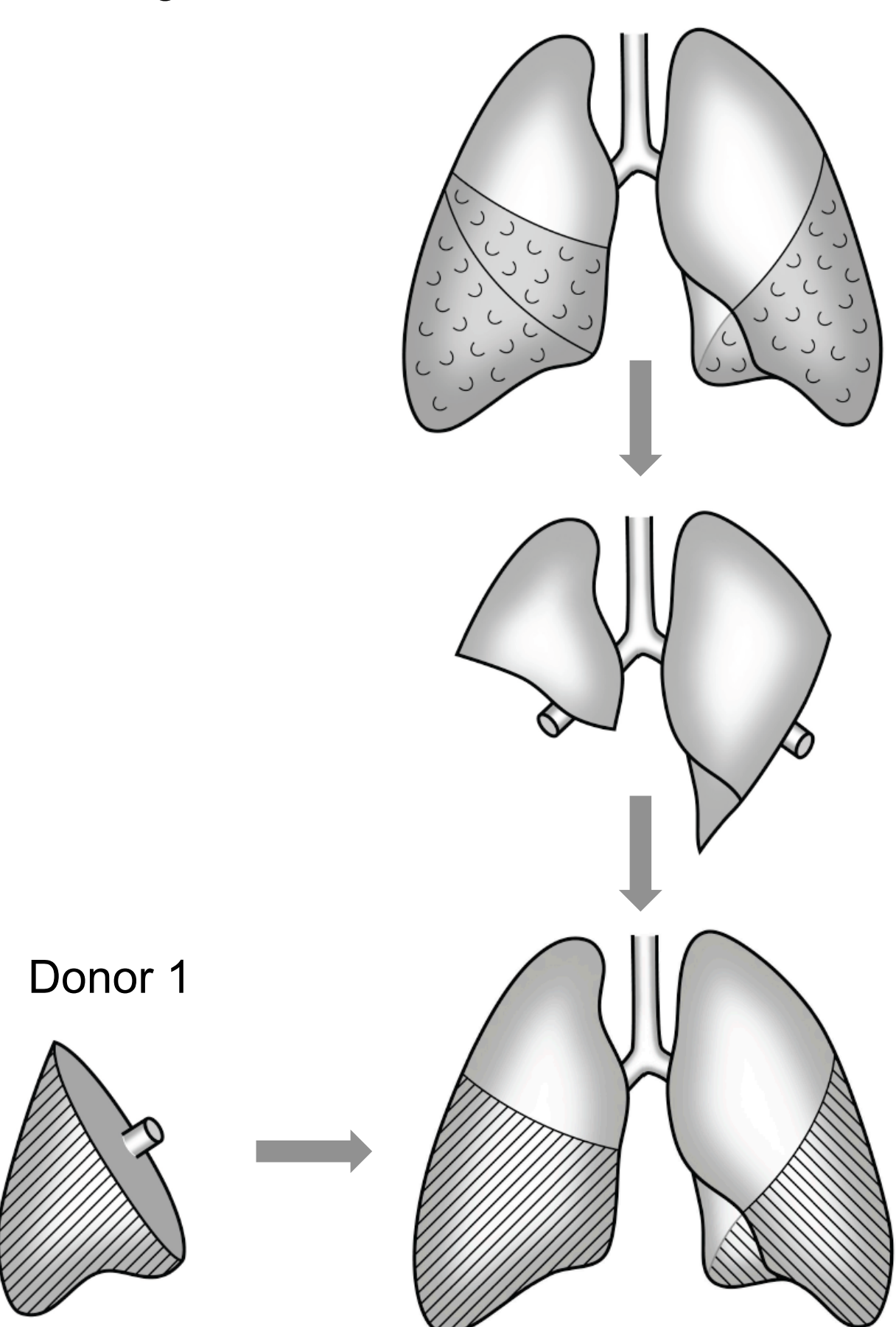

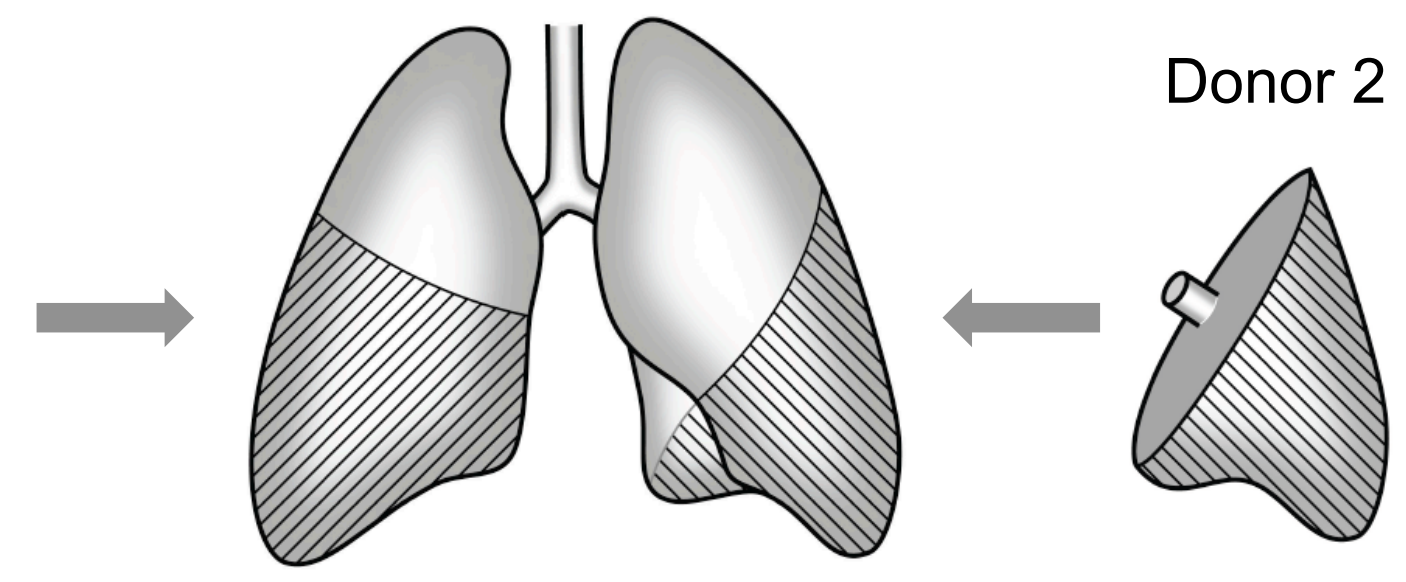

Donor 2

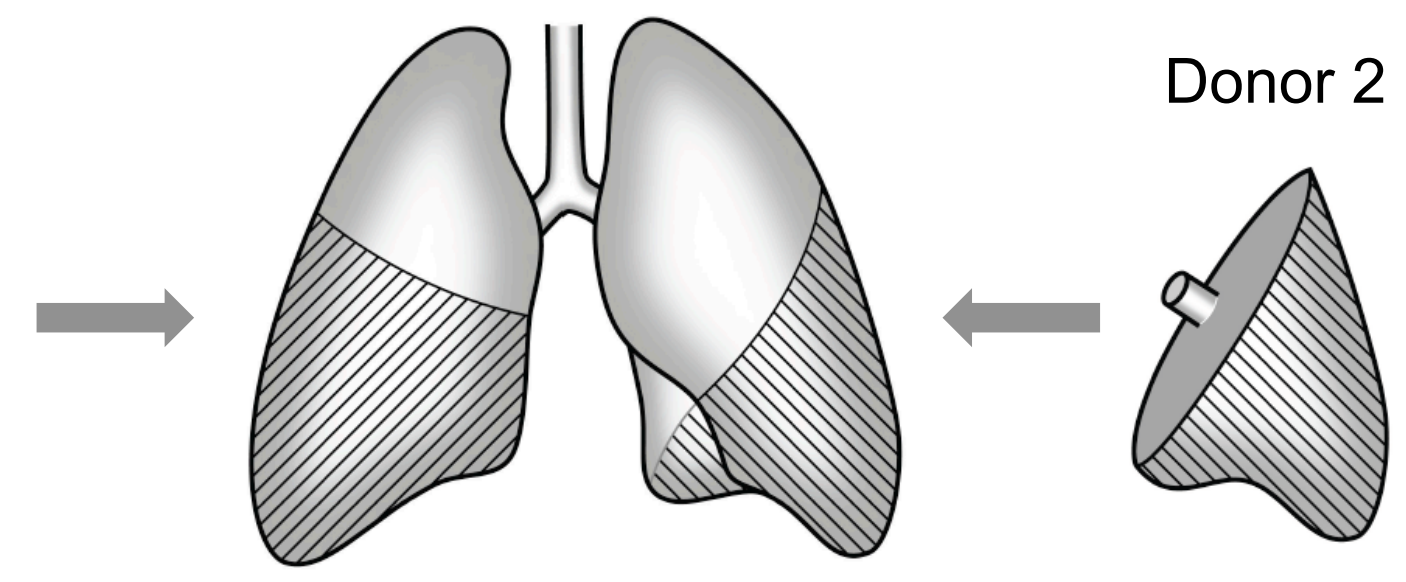




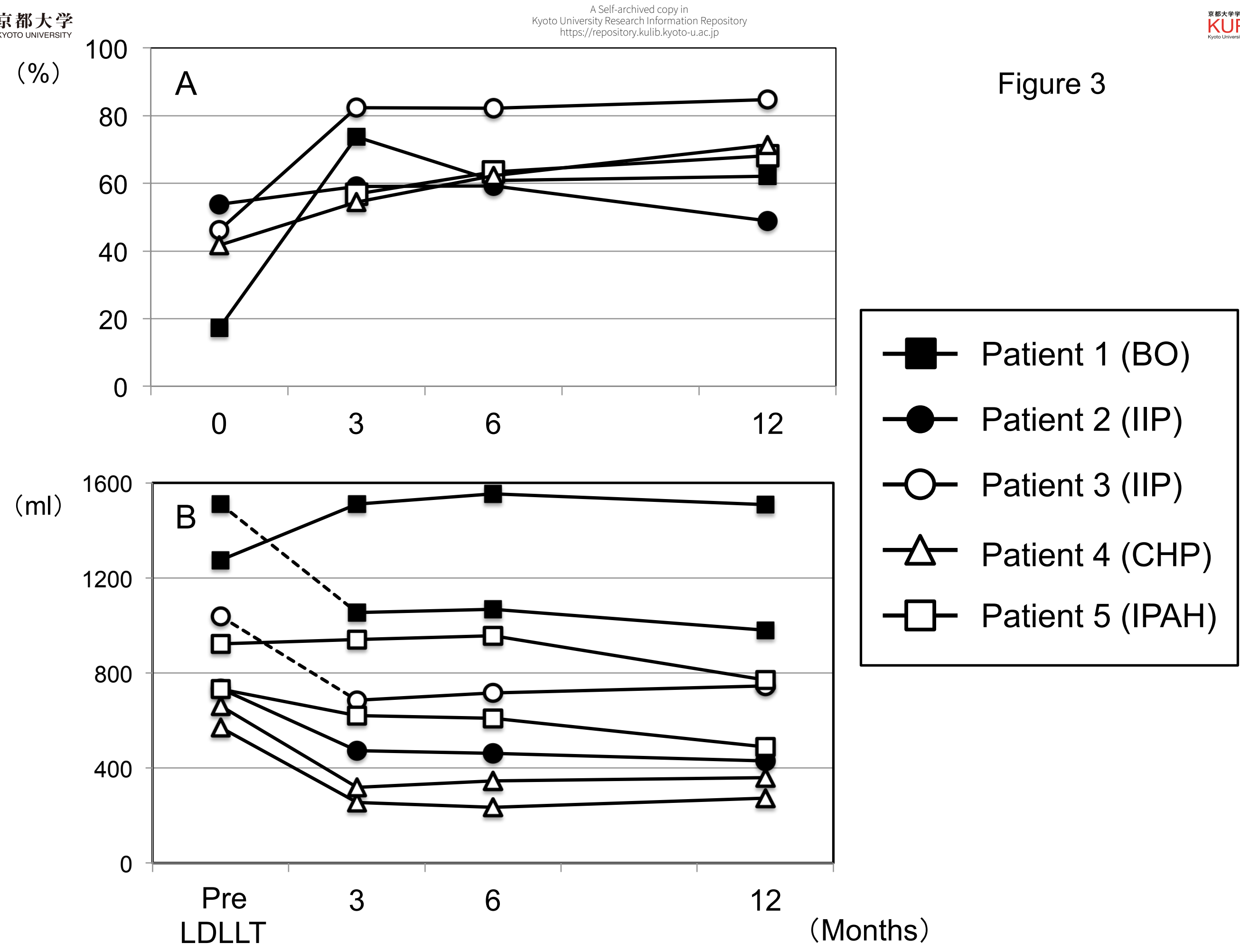


Figure 4

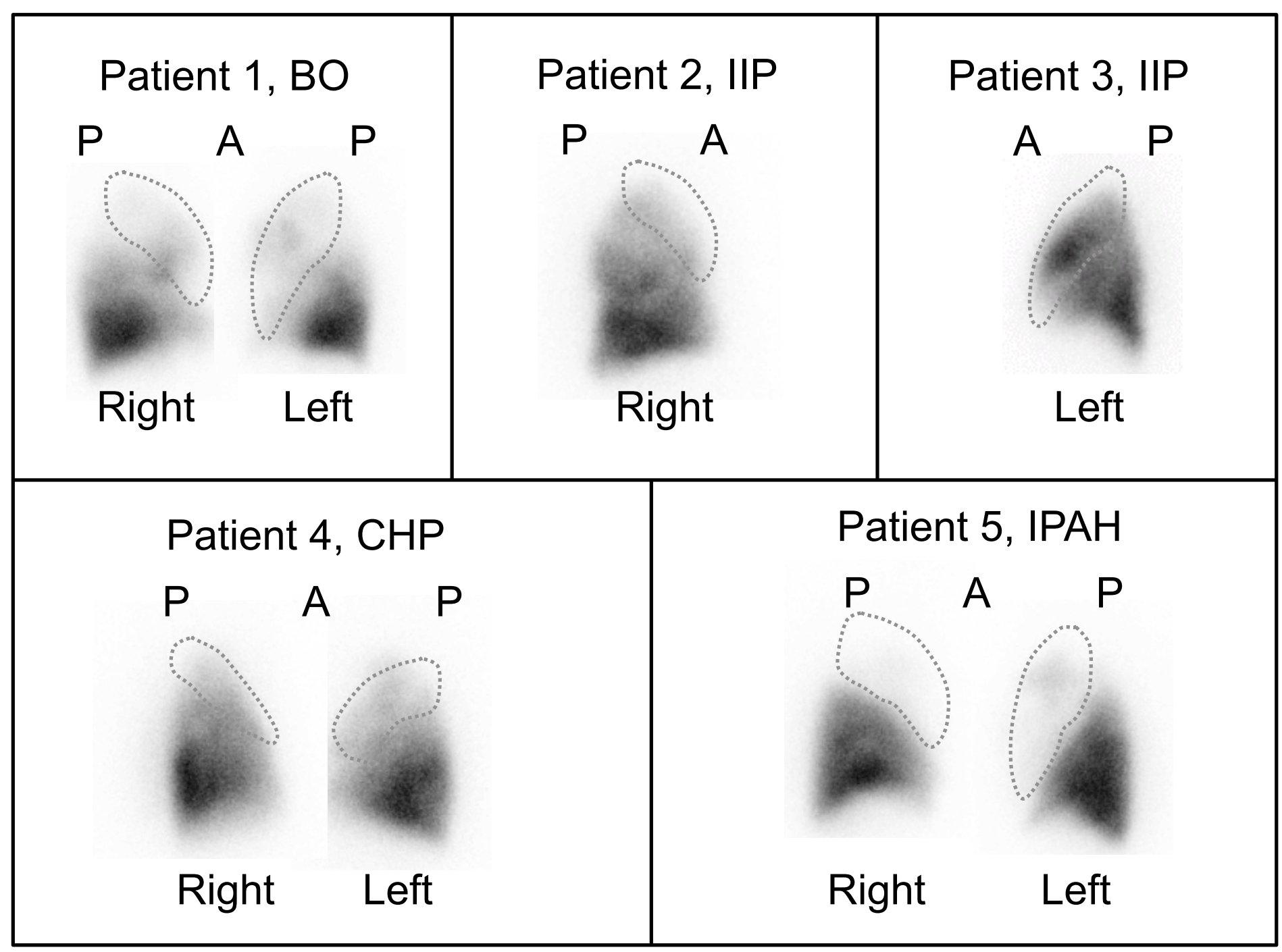

